\title{
Histology and Type I Collagen, Tenascin and Elastin Expression in Autologous and Allogeneic Anterior Cruciate Ligamentoplasties
}

\author{
I. Guillén-Vicente, J. M. López-Alcorocho, J. Salvador-Marín, E. Rodriguez-Iñigo, \\ G. Díaz-Motola, T. F. Fernández-Jaén, E. Ibáñez, P. Guillén-García
}

Traumatology and Orthopedic Surgery Department and Research Unit, CEMTRO Clinic, Madrid, Spain

\author{
CORRESPONDING AUTHOR: \\ Juan Manuel López-Alcorocho \\ Research Unit \\ CEMTRO Clinic \\ Ventisquero de la Condesa Street 42 \\ 28035 Madrid, Spain \\ E-mail: jm.lopez@amplicel.com \\ DOI: \\ 10.32098/mltj.03.2020.17
}

LEVEL OF EVIDENCE: 4

\begin{abstract}
SUMMARY
Background. Ligamentization has been accepted in Anterior Cruciate Ligament (ACL) surgery. The purpose of this study is to evaluate ligamentization of different allografts and autografts used as plasties through histological and type I collagen (COL1), tenascin (TEN) and elastin (ELA) expression analysis.

Methods. Prospective study of patients who underwent ACL reconstruction with at least 1-year follow-up. Biopsies were taken by arthroscopy and tissue sections were stained with hematoxilin-eosin and Masson's trichrome. Relative COL1, TEN and ELA expression was studied by RT-PCR. Hamstring tendon and intact ACL biopsies were used as controls.

Results. Eleven patients with a mean age of $36.6 \pm 12.0$ years were included. In 9 cases, ACL reconstruction was carried-out with autologous hamstring tendons while in the 2 remaining patients ACL was reconstructed using an achilles allograft. COL1, TEN and ELA expression of plasties was significantly different to ACL but similar to hamstring tendon. Histological analysis showed a dense fibroconnective tissue with cells similar to tenocytes, which could not be classified neither as a tendon nor as a ligament.

Conclusions. An ACL plasty, independently of its origin (autologous hamstring or allogeneic achilles) does not become a ligament, at least in our study period ( 5 years) but it does maintain ACL functionality.
\end{abstract}

KEY WORDS

Anterior cruciate ligament; hamstring autologous graft tendon; achilles allogenous graft tendon; histological analysis; ligamentization; tenocites.

\section{BACKGROUND}

Therapy for anterior cruciate ligament (ACL) tear includes invasive surgical techniques, in which the ruptured ACL is replaced by autologous (patellar, quadricipital or hamstring more frequently) or allogeneic tendons (1). The objective of surgery is to restore knee biomechanics by reproducing the mechanical conditions of the native ACL. However, the original biological structures are substituted by different ones: a tendon replaces a ligament.

Tendons and ligaments are elastic connective tissues with similar composition but different arrangement of their components, which is related to the different roles they have to perform in the musculoskeletal system: ligaments connect bones to each other in order to restrict their relative motions and tendons link muscles to bones (2). Water is the main component for both structures $(55-70 \%)$ but exists in a higher percentage in tendons (2). Type I collagen, which represents $70-80 \%$ of the dry weight (higher in tendons), is responsible for the high tensile strength and hierarchical structure $(3,4)$. Proteoglycans, glycosaminoglycans and glycoproteins are other molecules present in ligaments' and tendons' extracellular matrix (ECM) (4). Fibroblasts (called tenoblasts in the tendon), embedded in the ECM, are the main cell type of tendons and ligaments and synthesize all ECM molecules $(2,3)$. All these components are hierarchically organized and while in tendons, fibers are present as compact parallel bundles, in ligaments they are compactly packed and not arranged in parallel bundles (5). 
It is commonly accepted that implanted tendon structures adopt the role of ligaments: However, intra-articular "ligamentization" of the graft does not imply a complete transformation of a tendon graft into a ligament. In literature, a biological transformation process has been described as the graft remodels itself into a viable ACL-like tissue (6-8). Authors refer to an early phase with central graft necrosis and hypocellularity until the fourth week, followed by a proliferation, remodeling and revascularization phase up to the $12^{\text {th }}$ week, followed finally by a restructuring phase which makes the graft resemble the properties of $\mathrm{ACL}^{3}$. Collagen fibers begin to resemble an ACL between 6 and 18 months after reconstruction ${ }^{9-11}$. However, although matured tendon graft looks macroscopically similar to intact ACL, several authors have shown differences in biological and mechanical properties as well as in the distribution and diameters of collagen fibers comparing hamstring autograft with intact ACL $(7,8,12,13)$. Some studies in animals have shown a histological structure halfway between tendon and ligament (13).

The purpose of this study is to evaluate the features of autologous hamstring and allogeneic achilles tendons used as plasty for the ACL reconstruction through gene expression, histological, cellularity and pathological analysis of the plasty structure compared to normal hamstring tendon and intact ACL.

\section{MATERIAL AND METHODS}

\section{Patients}

This is a prospective case-series study carried out in patients operated for ACL reconstruction more than 1 year ago and who had been admitted to undergo new surgery in the same knee for other cause different to graft failure. The study was performed in accordance to ethical standards of the Helsinki Declaration of 1964, revised in 2013 and approved by the Institution's Research Committee. All included patients signed an informed consent (14). Eleven patients accepted to participate in the study and a biopsy of the graft was taken during the new surgery. During exploration all patients showed stable knees. New surgeries consisted in total knee arthroplasty (1 case), arthroscopy after a sport injury (4 cases), meniscectomy (4 cases) and autologous chondrocyte implantation ( 2 cases). In the surgery, plasty integrity was confirmed in all cases. In order to take the plasty biopsy, synovial membrane that covered the plasty was firstly removed. Then, a1-2 $\mathrm{mm}$ biopsy was taken in the middle third of the plasty with an arthroscopy basket forceps. Eight of these eleven patients accepted for a biopsy from hamstring tendon to be taken at the same surgical act. In the study, 3 more patients who were due to undergo a knee surgery for meniscal lesions were asked for a biopsy of the ACL to be taken to provide an intact ACL as a control. Biopsies were also taken in the middle third of the ligament following the same protocol described above. At the surgery moment, these patients showed stable knees and no signs of osteoarthrosis were observed in any case.

\section{Histological analysis}

One portion of each biopsy was stored at $-20^{\circ} \mathrm{C}$ in RNAlat$\mathrm{er}^{\mathrm{TM}}$ Stabilization Solution (ThermoFisher Scientific, Alcobendas, Spain) for gene expression study. The remaining fragment was fixed in formalin and paraffin-embedded. Blocks were cut using a microtome into $4-\mu \mathrm{m}$-thick sections. Tissue sections were stained with hematoxilin-eosin and Masson's trichrome staining to study both tissue architecture and collagen composition as well as fiber disposition.

\section{Gene expression analysis}

RNA was isolated after treatment with TRIzol (ThermoFisher Scientific) and chloroform and isopropanol precipitation. One $\mu \mathrm{g}$ RNA was reverse-transcribed using Oligo(dT) (ThermoFisher Scientific), RNasin (Promega Co, Madison, WI) and the SuperScrit ${ }^{\circledR}$ Reverse Transcriptase kit (ThermoFisher Scientific), following manufacturers' instructions. Relative expression of type I collagen (COL1); GenBank accession: NM_000088.3, tenascin-C (TNC); accession: NM_002160.3, and elastin (ELA); accession: NM_000501.3, genes was determined by real-time PCR (RT-PCR) in a StepOnePlus thermocycler (Life Technologies, Alcobendas, Spain) using the expression of the $\beta$-actin (ACT) housekeeping gene; accession: NM_001101.3; as standard. Each gene was amplified using the specific primers and probes commercially designed (Taqman Gene Expression Assays, Life Technologies). All amplifications were carried-out using the Taqman Universal PCR Master Mix (Life Technologies), following the manufacturer's instructions. Each sample was tested in triplicate and all genes were studied in the same PCR run.

\section{STATISTICAL ANALYSIS}

Statistical analysis was performed using the IBM SPSS Statistics version 22 software. Qualitative variables were expressed as a count and/or percentage. Normality of quantitative variables was checked with the Shapiro-Wilk test and expressed as the median (minimum - maximum) (non-normal variables) or mean \pm standard deviation. Intraclass correlation coefficient (ICC) and 95\% confidence interval for ICC were estimated to check consistency of trip- 
licate measure corresponding to COL1, TNC and ELA gene expression. Chicchettti and Domenic's criteria $a^{15}$ were used to interpret reliability of measures for ICC value. According to these authors, consistency was considered poor if ICC value was less than 0.40; fair if it was between 0.40 and 0.59 ; good if the value was between 0.60 and 0.74 and excellent if ICC value was between 0.75 and 1.00 .

With respect to COL1, TNC and ELA relative expression in ACL plasties, hamstring tendon and healthy ACL, if normality could not be demonstrated, expression of each gene were to be expressed as the median (minimum - maximum) of the triplicate and distribution in each sample type (plasty, hamstring tendon and ACL) and then contrasted with Mann-Whitney's U test (2 variables) or the Kruskal-Wallis test, including pairwise post-hoc tests (more than 2 variables). If variables had normal distributions, gene expression were to be expressed as the mean \pm standard deviation. Student's t test ( 2 variables) or ANOVA were used to contrast gene expression means, including pairwise post-hoc tests carried-out with Tukey's test (more than 2 variables). Gene expression distribution among the different causes second operation was checked with the Kruskal-Wallis test. Spearman's coefficient was used to study the existence of correlation.

Categorical variables were expressed as counts and/or percentage and compared with the Chi-Square Test, if applicable.

In all comparisons and parameter estimation, a value of $\mathrm{p}<0.05$ (two-sided) was considered statistically significant.

\section{RESULTS}

Eleven patients ( 11 knees) were included in the study. Nine of them were men and 2 of were women with a mean age of $36.6 \pm 12.0$ years. Six ligamentoplasties were performed in right knees and 5 in left knees. In 9 cases, ACL reconstruction was carried out with autologous hamstring tendons (semitendinosus and gracilis) while in the 2 remaining patients ACL was reconstructed using an achilles allograft (figure 1). All patients practiced non-professional sport (7 soccer, 2 horse riding, 1 ski and 1 paragliding). Elapsed median time between ACL ligamentoplasty and new surgery was 4.0 years (2-30 years). As a control, healthy ACL samples were taken from 3 patients (two men and 1 woman who were 32, 34 and 41) all operated arthroscopically for meniscal lesions.

COL1, TNC and ELA relative expression was measured in triplicate. In table I, ICC and $95 \%$ confidence interval of ICC is depicted. For the 3 genes ICC value was between 0.40 and 0.59 and thus, the relative expression estimation of each gene by means of the triplicate measures was considered fairly consistent. COL1, TNC and ELA relative expression in ACL plasties, hamstring tendons and healthy ACL were compared (figure 2). For each gene we found that means showed statistically significant difference among the three samples $(\mathrm{p}<0.001$ in each case, ANOVA). Pairwise comparisons gave similar results in the 3 genes: COL1 and TNC expression showed statistically significant higher values in the plasties and hamstring tendons than in healthy ACL while the opposite occurred with ELA expression which was significantly higher in healthy ACL than in plasties and hamstring tendon. In both situations, no statistically significant differences existed between plasty and hamstring tendon gene expression. To study the influence of the environment, gene expression was compared among the causes for the second operation, No significant differences were found between gene expression and cause for second surgery in any of the three studied genes $(\mathrm{p}=0.151$ for COL1, $\mathrm{p}=0.247$ for TNC and $\mathrm{p}=0.248$ for ELA; Kruskal-Wallis test). No correlation was found between COL1, ELA and TEN expression and elapsed time until second surgery either. Finally, we compared gene expression of patients implanted with autologous hamstrings with those implanted with allograft achilles tendon and no differences were found (figure 3).

Tissue architecture and collagen fibers disposition studied by hematoxylin-eosin and Masson's trichrome staining showed a dense fibroconnective tissue in all biopsies. In the case of control healthy hamstrings a collagenized matrix with a low-frequency crimp pattern of the collagen fibers is observed. Scattered in the collagen matrix, spindle-shaped cells similar to fibroblasts can be observed. Normal ACL tissue showed a similar fibroconnective tissue but with a high-frequency crimp pattern of the collagen fibers and similar spindle-shaped fibroblasts among collagen fibers. ACL grafts showed a dense fibroconnective tissue with a frequency crimp pattern of the collagen fibers intermediate between normal healthy hamstrings and ACL controls (figures 4,5). Spindle-shaped and ovoid fibroblasts disposed throughout collagen fibers were also observed. Although no differences in tissue organization between hamstrings (autologous) and achilles (allogeneic) grafts were observed, cellularity increased in the 5 -year evolution grafts (figure 5) with respect to those of 2-year evolution (figure 4), independently of graft nature. Five-year evolution tissue showed more vascularization than two-year biopsies. No histological differences have been found when the causes for the second surgery were compared. Histologically, in all cases tissue graft showed a tissue organization which could be classified neither as a tendon nor as a ligament (figures 4,5 ). 


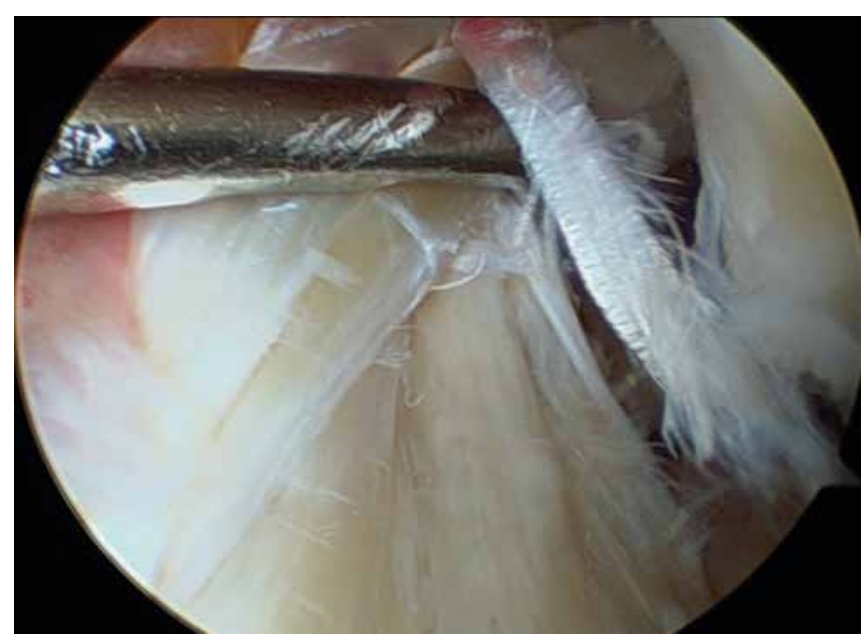

Figure 1. $A C L$ reconstruction, 8 years later. Arthroscopic view.

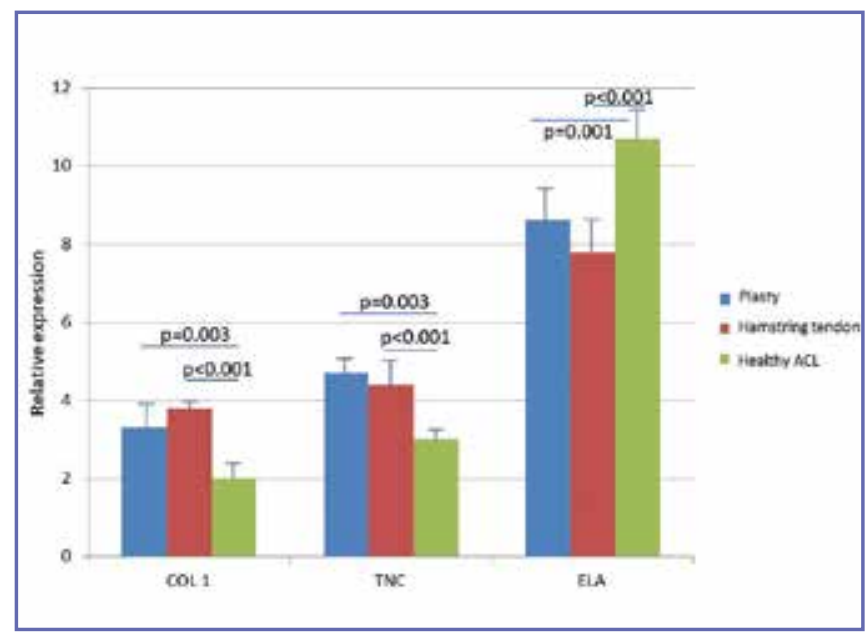

Figure 2. Mean relative Type I Collagen (COL1), tenascin (TNC) and elastin (ELA) expression in plasties, hamstring tendon and healthy ACL. Mean expression of these genes was significantly different among tissues ( $p<0.001$; ANOVA).

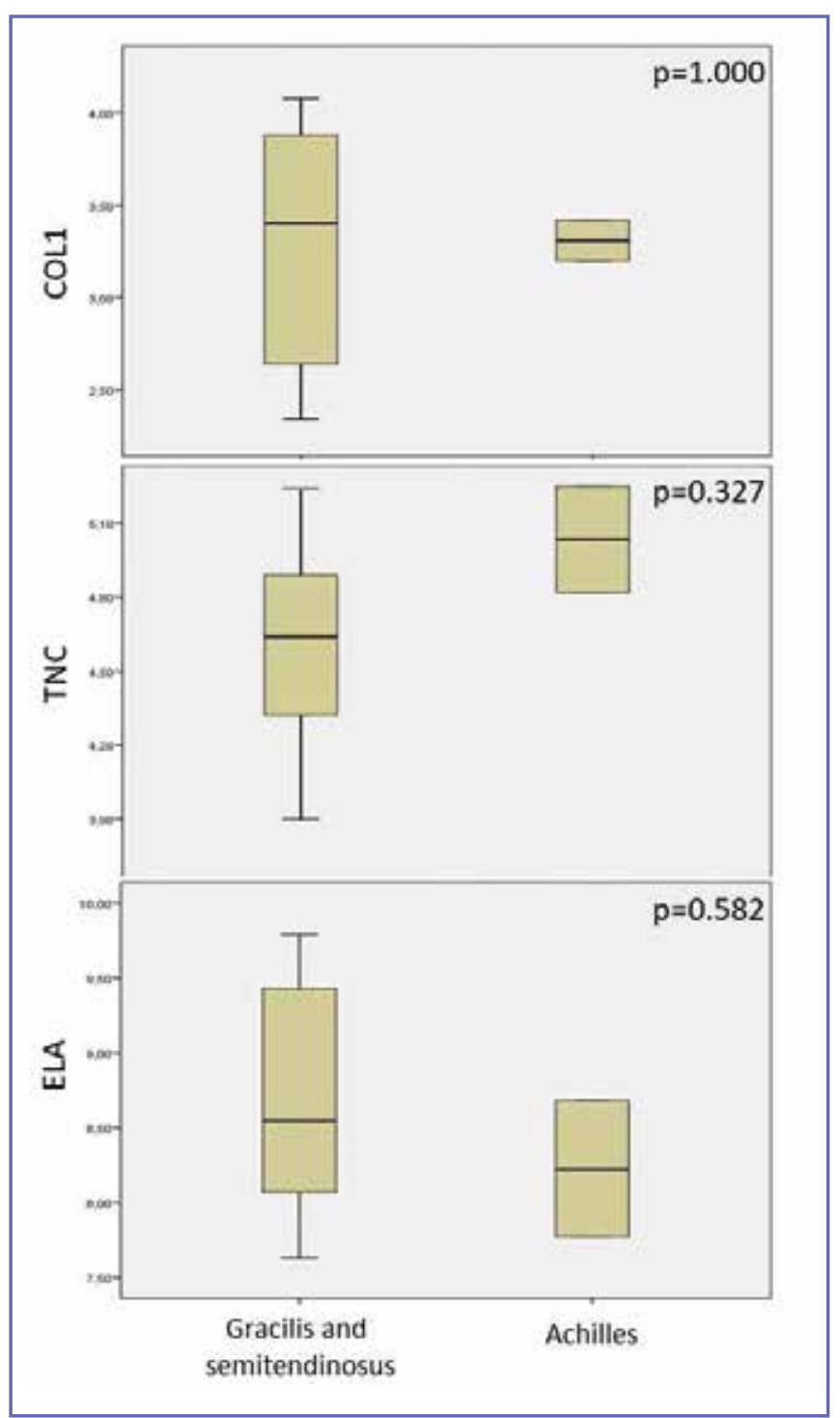

Figure 3. COL1, TNC and ELA expression in autologous hamstrings or allogeneic Achilles tendon plasties. Statistical comparisons were carried-out with the Mann-Whitney's U test.

Table I. Intraclass Correlation Coefficient (ICC) and 95\% confidence interval of ICC for relative Type I Collagen (COL1), tenascin-C (TNC) and elastin (ELA) expression.

\begin{tabular}{llll}
\hline & COL1 & TNC & ELA \\
\hline ICC & $\begin{array}{l}\text { (95\% Confidence Interval) } \\
\text { (-0.121-0.783) }\end{array}$ & $\begin{array}{l}0.493 \\
(-0.089-0.789)\end{array}$ & 0.548 \\
\hline -value & 0.048 & $0.029-0.812)$ \\
\hline
\end{tabular}



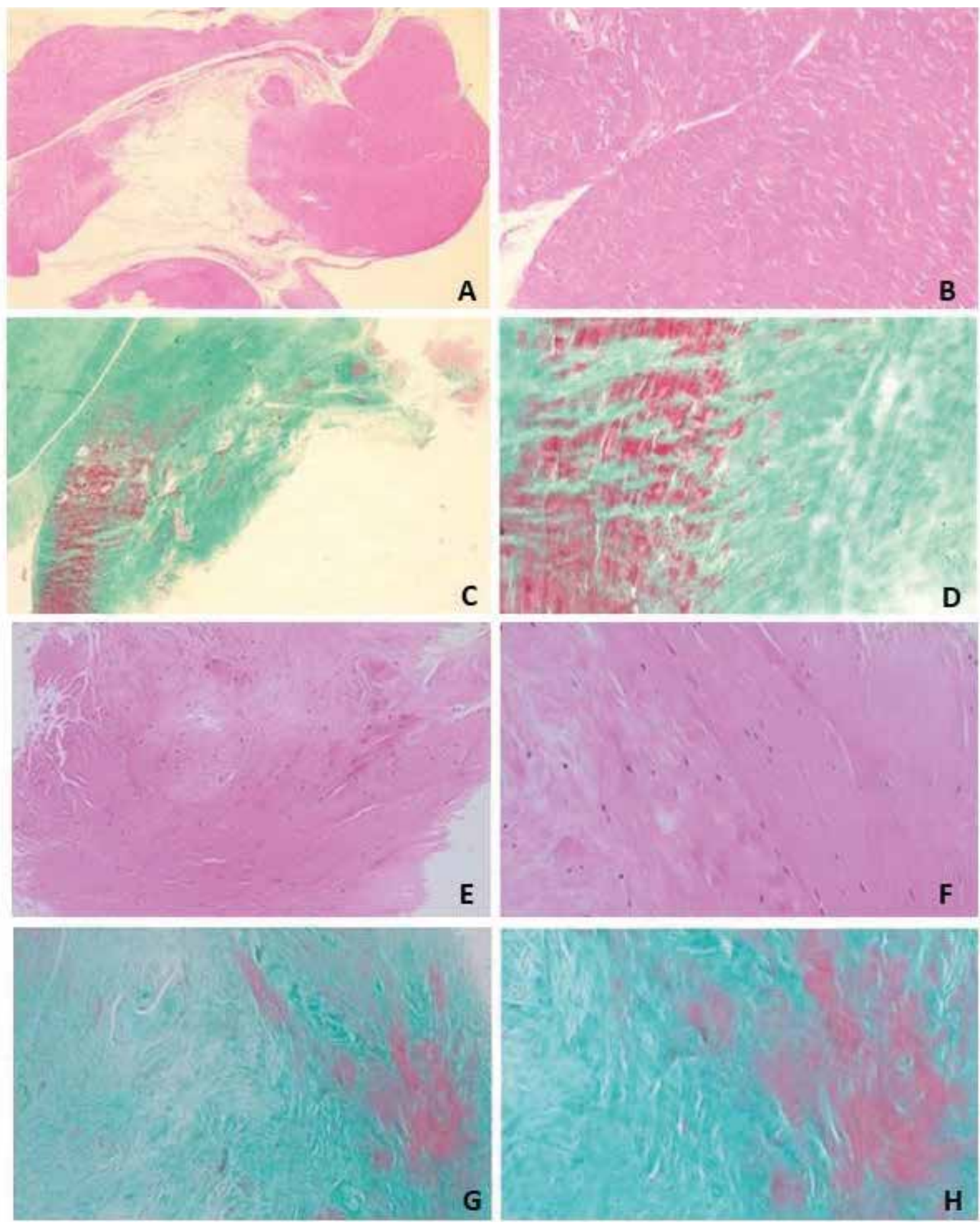

Figure 4. Representative pictures of hematoxilin-Eosin and Masson Trichrome staining of two-year (A-D) and three-year-evolution $(E-H)$ hamstring tendons plasties. Collagenized fibroconnective tissue with the presence of spindle-shaped and ovoid fibroblasts scattered in the collagen matrix and focal smooth muscle fibers is observed. A and E: Hematoxilin-Eosin staining 4x; B and F: Hematoxilin-Eosin staining 20x; C and G: Masson's trichrome 4×; D and H: Masson's trichrome 20x. 

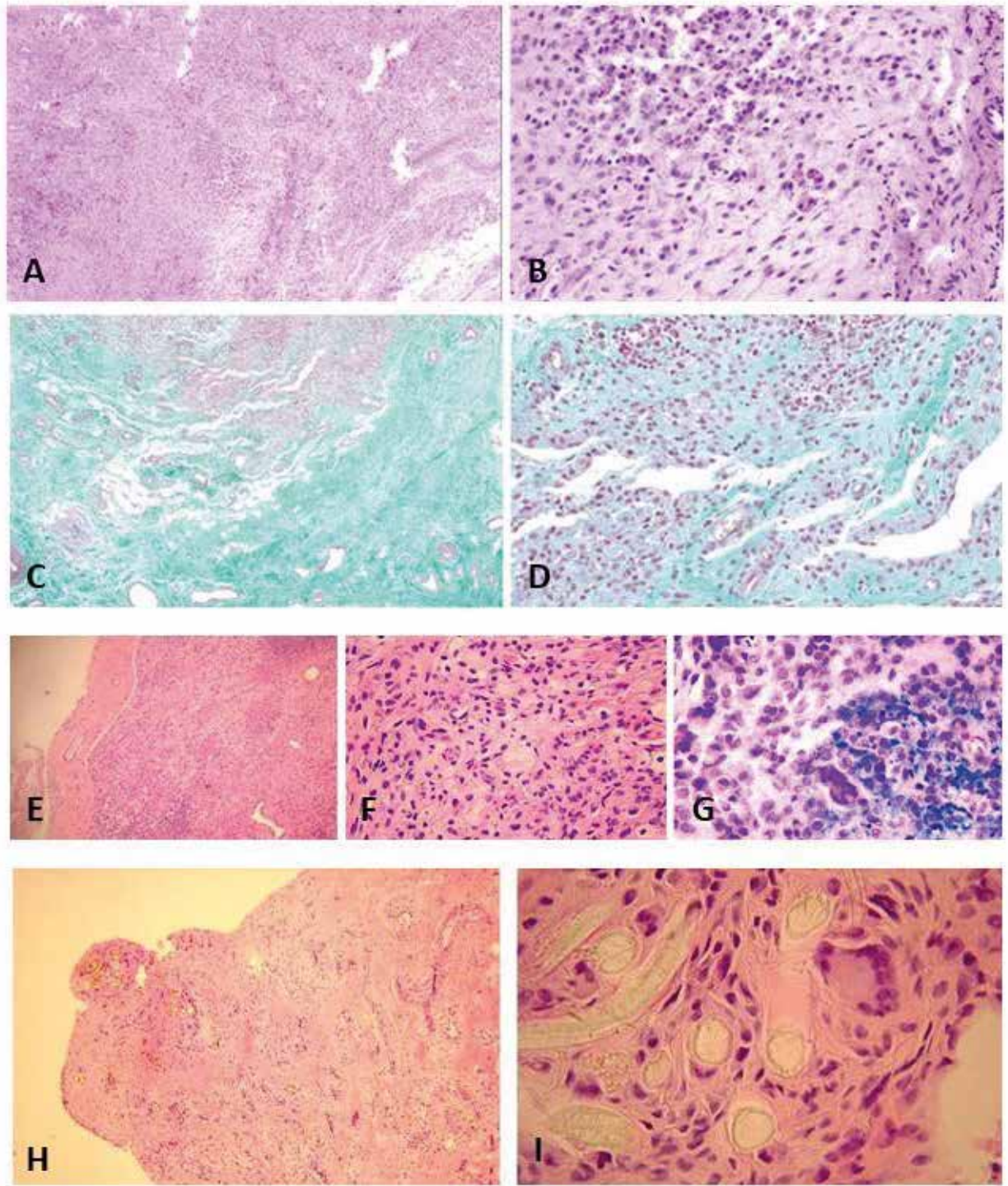

Figure 5. Representative pictures of five-year evolution autologous hamstring (A-D) and allogeneic achilles tendons plasties (E-I). In the hamstring tendon plasty, a collagenized fibroconnective tissue with spindle and ovoid cells and the presence of smooth muscle fibers is observed (A-D). The other two samples corresponded to achilles allograft tendon plasties (E-I) and a collagenized fibroconective tissue with similar spindle-shaped aand ovoid cells is shown. Notice the presence of vascular structures in both autologous and allogeneic grafts . A,E,H: Hematoxilin-Eosin staining 4x; B,F,l: Hematoxilin-Eosin staining 20x; C: Masson's trichrome 4x; D: Masson's trichrome 20x; G: Hematoxilin-Eosin staining 40x.

\section{DISCUSSION}

In this work we try to answer the question: is tendon a good substitute for an anterior cruciate ligamentoplasty? Hamstring autologous and Achilles allogeneic tendon plasties did not become a similar tissue to native ACL. Some authors observed tendon-specific biological features which did not exhibit ligamentous histological properties and for this reason the studied tissue resembles a tendon more than a ligament. Authors agree that the graft will never be completely transformed into normal ACL tissue $(7,10,16)$, although it could exhibit similar biomechanical properties when it comes to restoring knee stability early, with a ratio of between $72 \%$ and $100 \%$ returning to sport ${ }^{17}$. In the presence of adequate surgical techniques and good rehabilitation programmes, failures and re-injury rates are relatively uncommon (3-19\%) $(18,19)$, which evidence there is still margin for improvement. 
In the present work, we have studied histological features and expression profile of some genes expressed in tendons and ligaments in ACL plasties from two different origins (autologous hamstrings and allogeneic achilles tendons) and with different evolution times, compared to normal hamstring tendon and intact ACL (8). In a systematic revision carried-out by Pauzemberg et al. (17), without including studies with allogeneic tendons, authors concluded that surgical technique and specially an anatomically incorrect reconstruction of the ACL could influence postoperative remodeling despite a possible increase of laxity or non-anatomical tension properties. It was not the case in our case-series, since $100 \%$ of included patients were operated by other causes.

In order to elucidate whether studied tissue was a tendon or a ligament, COL1, ELA and TEN were chosen due to the different expression pattern in both tissues. In fact, it has been published that COL1 and TEN are more abundant in tendons while ELA presents higher values in ligaments $(4,5)$. Analysis of COL1, ELA and TEN in plasties revealed an expression pattern closer to tendon than to ligament, with higher mean amount of COL1 and TEN mRNA in tendons and plasties than in ligaments and higher mean expression of ELA in ligaments than in tendons and plasties $(4,5)$, independently of plasty nature and the time elapsed between ligamentoplasty and sample harvesting. At least, with respect to the three studied proteins, these results suggest that both autologous and allogeneic plasties continue having the same protein expression profile than tendons. As far as we know, no studies about protein expression of ligamentoplasties in humans have been published. Most studies have been performed in animal models, especially in rabbits (20). Xie et al. (21) studied the expression pattern of COL1, COL3, growth factors, angiogenesis-promoting, and nerve-related genes in rabbits, concluding that ligament pattern expression is maintained when ACL remnant is preserved during ACL reconstruction. In our case, tibial fibers remnant are not used in the plasties, which could explain that expression of targeted proteins was more similar to tendon than to ligament.

As stated above, from a histological point of view, tendons and ligaments are structurally quite similar but they differ at the ultrastructural level, being the amount and disposition of large-diameter collagen fibers and collagen fibrils (8) and crimp pattern frequency of the collagen fibers $(22,23)$ the main differences between both tissues. Crimp pattern frequency of the collagen fibers is highly correlated with the presence of myofibroblasts, a specialized cell type involved in crimp formation, whose number is increasing during graft evolution time. On the other hand, Abe et al. (23) have demonstrated that during ACL remodelation, crimp pattern frequency is gradually changing and vascularization is increasing along timeOur plasties had a collagen crimp pattern frequency intermediate between tendon and ligament and higher cellularity and vascularization in 5-year evolution plasties compared with the 2-year ones. Our results support plasty remodeling after ACL replacement by a tendon, becoming a tissue whose features are intermediate between tendon and ligament. Since no differences were found between autologous hamstrings or allogeneic achilles tendon plasties, similar ligamentization process may occur irrespectively of graft nature.

Main weakness of the study are sample size and a relatively short histological determination. Its main strengths are the comparison with healthy hamstring tendon and ACL. This work represents the first attempt to determine protein expression together with histological study of ACL plasties from different origins: autologous hamstring and allogeneic achilles tendons. Furthermore, in the study, plasties from 2 to 5 -year evolution have been included. However, a more detailed study, including relative expression of a large set of genes, including those involved in angiogenesis, vascular and nerve regeneration, tissue growth and more detailed histological study including other techniques such as light microscopy with polarization and electron microscopy is now been planned with the aim of ascertaining the mechanisms involved in the process commonly known as ligamentization.

\section{CONCLUSIONS}

Taken together, our results suggest that an ACL plasty, independently of its origin (autologous hamstring or allogeneic achilles tendon) does not become a ligament in a period of 5 years, although it is able to maintain ACL functionality and contribute to knee stability. Protein expression and histological analysis reveal that in the course of several years, plasties are gradually remodeled and become an intermediate tissue somewhere between a tendon and a ligament. This tissue shows very similar COL1, ELA and TEN expression patterns to tendons but typical collagen fiber disposition leans more on the side of a ligament, with time lapse being irrelevant.

\section{ACKNOWLEDGMENTS}

The authors gratefully acknowledge Mario Wensell for carefully reviewing the manuscript's linguistic. This work has been supported by a grant from the Fundación Dr. Pedro Guillén. 


\section{AUTHOR CONTRIBUTIONS}

IGV: ligamentoplasties, biopsies harvesting, manuscript reviewing; JMLA: gene expression by RT-PCR, statistical analysis, manuscript written; JSM: analysis of results, manuscript written; ERI: histological analysis, manuscript reviewing; GDM: analysis of results, manuscript reviewing; EI: ligamentoplasties, biopsies harvesting; TFFJ: ligamentoplasties, data analysis, manuscript reviewing; PGG: ligamentoplasties, biopsies harvesting, study planning, manuscript reviewing

\section{CONFLICT OF INTERESTS}

The authors declare that they have no conflict of interests.

\section{ETHICS}

The authors declare that this research was conducted following basic, ethical aspects and international standards as required by the journal (13).

\section{REFERENCES}

1. Marieswaran M. Jain I, Garg B, Shama V, Kalyanasundaram D. A Review on Biomechanics of Anterior Cruciate Ligament and Material for Reconstruction. Appl Bionics Biomech 2018: May 13. doi: $10.1155 / 2018 / 4657824$.

2. Hoffmann A, Gross G. Tendon and ligament engineering in the adult organism: mesenchymal stem cells and gene-therapeutic approaches. Int Orthop. 2007;31:791-7.

3. Mienaltowski MJ, Birk DE. Structure, physiology, and biochemistry of collagens. Adv Exp Med Biol. 2014;802:5-29.

4. Miller MD, Thompson SR. Miller's review of orthopaedics, $7^{\text {th }}$ Edition. Philadelphia: Elsevier 2012

5. Zitnay JL, Weiss JA. Load transfer, damage, and failure in ligaments and tendons. J Orthop Res. 2018;36:3093-104

6. Amiel D, Kleiner JB, Roux RD, Harwood FL, Akeson WH. The phenomenon of "ligamentization": anterior cruciate ligament reconstruction with autogenous patellar tendon. J Orthop Res. 1986;4:162-72

7. Zaffagnini S, De Pasquale V, Marchesini Reggiani L, et al. Electron microscopy of the remodelling process in hamstring tendon used as ACL graft. Knee Surg Sports Traumatol Arthrosc. 2010;18:1052-58.

8. Dong S, Xie G, Zhang Y, Shen P, Huangfu X, Zhao J. Ligamentization of Autogenous Hamstring Grafts after anterior cruciate ligament reconstrution : Mid-term versus Long-term Results. Am J Sports Med 2015;43:1908-17.

9. Weiler A, Peine R, Pashmineh-Azar A, Abel C, Südkamp NP, Hoffmann RF. Tendon healing in a bone tunnel. Part I: biomechanical results after biodegradable interference fit fixation in a model of anterior cruciate ligament reconstruction in sheep. Arthroscopy 2002; 18:113-23.

10. Abe S, Kurosaka M, Iguchi T, Yoshiya S, Hirohata K. Light and electron microscopic study of remodeling and maturation process in autogenous graft for anterior cruciate ligament reconstruction. Arthroscopy. 1993;9:394-405.

11. Claes S, Verdonk P, Forsyth R, Bellemans J. The "ligamentization" process in anterior cruciate ligament reconstruction: what happens to the human graft? A systematic review of the literature. Am J Sports Med. 2011;39:2476-83

12. Janssen RP, Scheffler SU. Intra-articulatr remodeling of Hamstring tendon grafts after anterior cruciate ligament reconstruction. KneeSurg Sports Traumatol Arthrosc 2014;22:2102-8.

13. Giordano M, Falciglia F, Poggiaroni A, Aulisa AG, Savignoni P, Guzzanti V. Histological changes of semitendinosus autograft after anterior cruciate ligament reconstruction in an immature rabbit model. J Exp Orthop.2015;2:17-25.

14. Padulo J, Oliva F, Frizziero A, Maffulli N. Muscles, Ligaments and Tendons Journal - Basic principles and recommendations in clinical and field Science Research: 2016 Update. Muscles Ligaments Tendons J. 2016:19;6:1-5

15. Cicchetti, Domenic V. Guidelines, criteria, and rules of thumb for evaluating normed and standardized assessment instruments in psychology. Psychological Assessment. 1994;6:284-90.

16. Cho S, Muneta T, Ito S, Yagishita K, Ichinose S. Electron microscopic evaluation of two-bundle anatomically reconstructed anterior cruciate ligament graft. J Orthop Sci. 2004;9:296-301.

17. Pauzenberger L, Syré S, Schurz M. "Ligamentization" in Hamstring Tendon Grafts After Anterior Cruciate Ligament Reconstruction: A Systematic Review of the Literature and a Glimpse Into the Future. Arthroscopy. 2013;29:1712-21.

18. Hui C, Salmon LJ, Kok A, et al. Fifteen-year outcome of endoscopic anterior cruciate ligament reconstruction with patellar tendon autograft for "isolated" anterior cruciate ligament tear. Am J Sports Med 2011;39:89-98.

19. Pinczewski LA, Lyman J, Salmon LJ, et al. A 10-year comparison of anterior cruciate ligament reconstructions with hamstring tendon and patellar tendon autograft: A controlled, prospective trial. Am J Sports Med 2007;35: 564-74.

20. Wu B, Qiu Z, Li S, et al. Histological properties of autogenous hamstring grafts after anterior cruciate ligament reconstruction. Zhongguo Xiu Fu Chong Jian Wai Ke Za Zhi. 2018;32:873-9.

21. Xie GM1, Huang Fu XQ, Zhao JZ. The effect of remnant preservation on patterns of gene expression in a rabbit model of anterior cruciate ligament reconstruction. J Surg Res. 2012;176:510-6.

22. Weiss M, Unterhauser FN, Weiler A. Crimp frequency is strongly correlated to myofibroblast density in the human anterior cruciate ligament and its autologous tendon grafts. Knee Surg Sports Traumatol Arthrosc. 2012;20:889-95.

23. Abe S, Kurosaka M, Iguchi T, Yoshiya S, Hirohata K. Light and Electron Microscopic Study of Remodeling and Maturation Process in Autogenous Graft for Anterior Cruciate Ligament Reconstruction. Arthroscopy. 1993;9:394-405. 